\title{
PREDICTIVE CREDITWORTHINESS MODELING IN ENERGY-SAVING FINANCE: MACHINE LEARNING LOGIT AND NEURAL NETWORK
}

\section{(iD) Herlan ${ }^{1}$ \\ (iD) Eka Sudarmaji ${ }^{2+}$ \\ (iD) M. Rubiul Yatim}

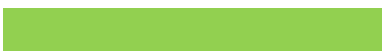

\section{Article History}

Received: 14 December 2021 Revised: 17 January 2022 Accepted: 31 January 2022 Published: 8 February 2022

\section{Keywords}

Creditworthiness

ESCO

Machine learning

Logit regression

LCCA

Retrofit finance.

\section{JEL Classification:} C25, C53, Q48.

\author{
${ }_{1,2,3}$ Faculty of Economics and Business, University of Pancasila, Jalan \\ Srengseng Sawah, Pasar Minggu Jakarta, Indonesia. \\ 'Email: herlan@univpancasila.ac.id Tel: 0816946278 \\ 'Email: esudarmaji@univpancasila.ac.id Tel: 087884964643 \\ ${ }^{s}$ Email:mrubiulyatim@univpancasila.ac.id Tel: 081384467762
}

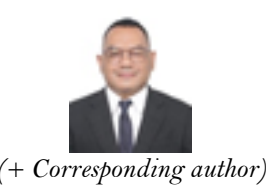

ABSTRACT

Customer's creditworthiness was becoming more crucial for ESCO. Machine learning was used to predict the creditworthiness of clients in retrofit financing processes. Machine learning was used to predict the creditworthiness of clients in ESCO financing processes. This research aimed to develop a retrofitting scoring model to leverage a machine learning and life cycle cost analysis (LCCA) to evaluate alternative financing for Energy Efficiency Saving in Indonesia. The model was built on the Logistic Regression model and Artificial Neural Networks model of machine learning. The model was developed and tested using the Python algorithm, and the proposed model's efficiency was demonstrated. The logistic regression calculations showed that the accuracy value of prediction data with test data was $88.3562 \%$ and $87.67 \%$ for Artificial Neural Networks and Logistic Regression models. The prediction rate result that refers to the correct predictions among all test data for Artificial Neural Networks and Logistic Regression model was $92.20 \%$ and $91.98 \%$, respectively. Meanwhile, the percentage of customers who were correct to all customers predicted to default was $94.41 \%$ for Artificial Neural Networks and $93.81 \%$ for the Logistic Regression model. Credit models were helpful to evaluate the risk of consumer loans. Finally, the quality and performance of these models were evaluated and compared to identify the best one. The logistic regression and neural network models obtained were good and very similar, although the neural network was slightly better.

Contribution/Originality: This study gained a deeper understanding of the obstacles in promoting energy efficiency practices in Indonesia's Building Energy Efficiency and ESCO. Therefore, the results of this study have implications for management science, management practices in the company and commercial building industry in Indonesia, and the government as a regulator.

\section{INTRODUCTION}

This research looked at the influence of an energy-efficiency program on one method of lowering energy consumption: switching to energy-saving lighting. The figure shows the example comparative measurement between LED energy-saving and conventional lightings shown in Table 1. Authors defined retrofits as the replacement of conventional lightings equipment with new LED energy-saving, or the construction of new infrastructure to increase energy efficiency and lower utility costs before the old equipment was damaged or reached the end of its economic life (Dobbs et al., 2013; Frankel, Heck, \& Tai, 2013; Husin, Ahmad, Ab Wahid, \& Kamaruzzaman, 2017; McWilliams \& Walker, 2005). Under this model, retrofit projects would need investment, and the worth of the retrofits would be determined by the investment payback time (Heesen \& Madlener, 2016; Kumbaroğlu \& Madlener, 2012). The value 
of the investment determined the payback time, energy expenses, and operational hours of the equipment (Angelis \& Nussbaum, 2015; Fuller \& Petersen, 1996; Ruparathna, Hewage, \& Sadiq, 2017). The operational hours of LED were used to determine the performance of the company's financial plans.

Table 1. Comparative measurement between LED and TLD.

\begin{tabular}{|c|c|c|c|}
\hline No & Description & TLD & LED TUBE \\
\hline 1 & Energy Consumption (Watt) & 72 & 16 \\
\hline 2 & Operational Hours & 8 & 8 \\
\hline 3 & Electricity Consumption/Kwh Year & 210.24 & 46.72 \\
\hline 4 & Electricity Bill/Kwh & 1.125 & 1.125 \\
\hline 5 & Electricity Cost in IDR & 236.520 & 52.560 \\
\hline 6 & Life Cycle in Hours & 10.000 & 15.000 \\
\hline 7 & Life Cycle in Years & 3 & 5 \\
\hline 8 & Lamp Price & 12.450 & 81.000 \\
\hline 9 & Differences in life cycle TLD vs. LED & 6.225 & - \\
\hline 10 & Energy cost/Year & 242.745 & 52.560 \\
\hline 11 & Difference Energy Saving TLD vs. LED & - & 190.185 \\
\hline 12 & Energy Saving in \% & & $78.35 \%$ \\
\hline
\end{tabular}

Two independent finance agreements were used for retrofitting: the Energy Saving Performance Contract (ESPC) and the Energy Saving Agreement (ESA). The key idea was that the ESPC and ESA stipulated that the client pays the ESCO from the savings in energy costs. The flow of duty, privileges, and the entity participating in the retrofitting finance plan is shown in Figure 1.

One of the reasons the authors investigated energy efficiency was technical improvements. In many regions, the employment of cutting-edge technology has created hurdles to embracing energy-saving energy technologies, resulting in inefficiencies in energy usage. Researchers speculated that sophisticated technology users could struggle to manage it Fornara, Pattitoni, Mura, and Strazzera (2016); Heesen and Madlener (2016); Stern (2011). All of these variables were converted into transaction costs. As a result, the authors understood how energy conservation works and the repercussions and how its creative business model, investment value, and new innovative financing choices would benefit everyone. Our research intends to develop a credit risk modeling methodology to lower the risk of credit granted to retrofitting projects while increasing ESCO benefits.

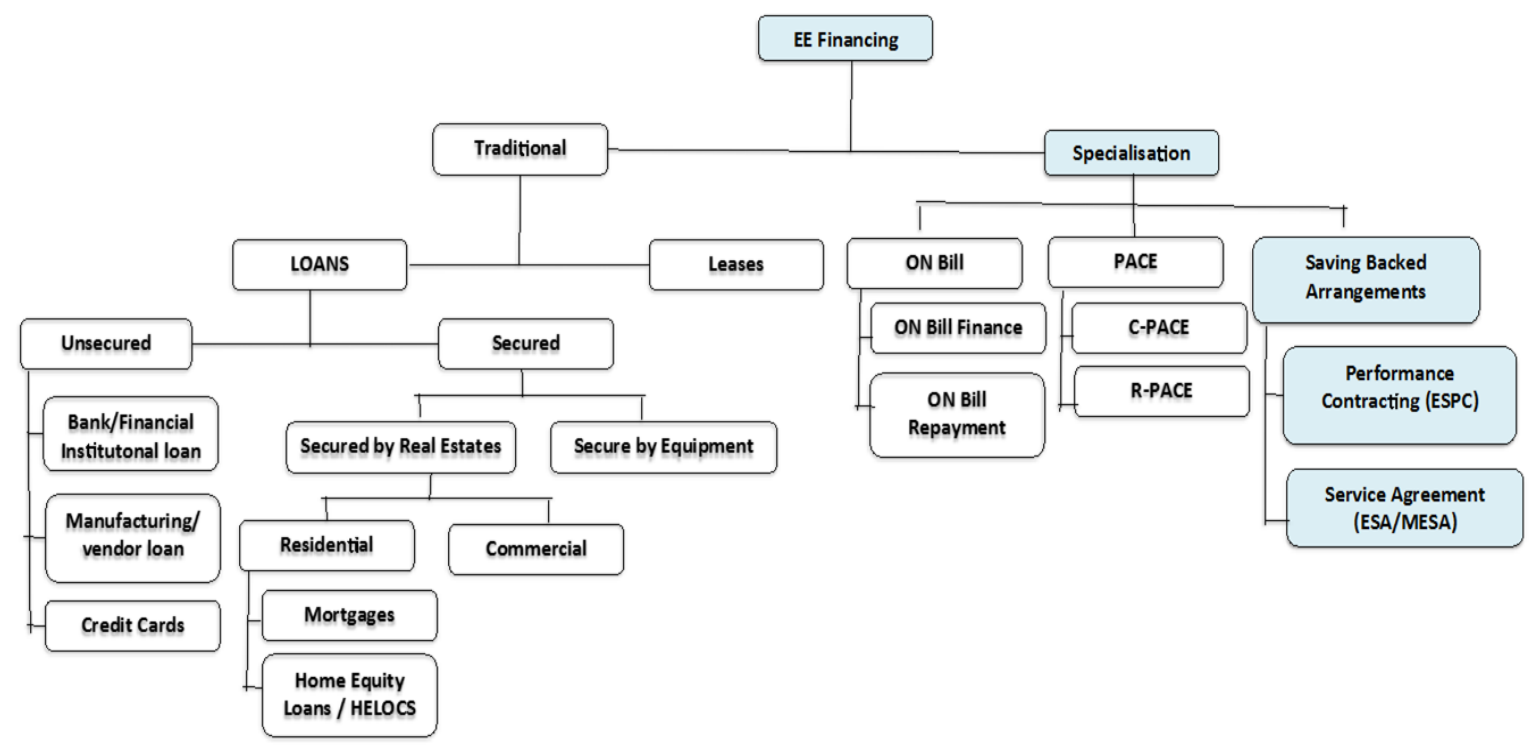

Figure 1. Energy efficiency financing (WSGR, 2015). 
Consequently, the goal of this research was to determine the optimum modeling with the highest performance and accuracy. The research employed the same methodologies and principles as EPA-Energy Start (The United States Environmental Protection, 2008) to assess the project's feasibility. The most important aspects to examine were the initial upfront investment, revenue factors, extra revenue factors, payback duration, investment returns, and client attributes. As a result, the firm's financial state was assessed first, followed by the return rate (payback). The hurdle rate was the agreed-upon criterion for passing the investment profitability test. If the IRR meets or exceeds predicted profits, the investment is profitable. A stream of future cash flows discounted with the project's risk provided the correct rate of return. As the cost of capital and risk grows, the amount of profit required would need more investment. Last but not least, the investor qualities must be taken into account. The approach was almost identical to banks' determining a customer's creditworthiness.

In addition to the cost of credit default risk, the cost of retrofitting aspects is an essential factor. The retrofitting process is the same as every other loan process. Financial selection criteria were generally measured through credit reports. Some of the problems that can be identified were 1) What the ML model can use variable determinants to predict the occurrence of retrofitting credit risk?, and 2) What is the right strategy to mitigate credit risk in the retrofitting service process at ESCO?. The paper seeks to demonstrate how ML can help ESCO cope with energy efficiency projects with real case studies. The paper investigates case studies conducted between June 2020 and January 2021. As a result, interview procedures were updated to obtain information directly from company directors, chief executives, and general managers. In this paper, the authors use a case study, where the ESCO provider was MWS, and the customer was SHJ' The authors refer to pseudonyms for the research. Until recently, MWS decided that credit for retrofitting projects was traditionally done, i.e., ensuring that customers met the criteria and covered all risks. Ultimately, ESCO management decided on a retrofitting project based on this individual analysis, where the final decision was made by acclamation based on this analysis. Here we designed a Retrofitting Risk Modelling System for ESCO using the ANN and LR model of ML. As a result, a sound credit risk modeling system could assist MWS in predicting credit risk.

Predictive analytics aims to build analytic models that predict the target size of interest. The target was used to direct the learning process during the optimization process. There are two types of predictive analytics depending on the size of the target, regression and classification. An example of a variable target in the regression was the creditworthiness of SHJ. As for the target, classification was a category, the binary classification between default and non-default. According to Halladay (2013), predictive analytics includes a variety of statistical techniques used for data analysis today, historically, economically, and even unstructured data such as sentence text and images. This information was used to identify some risks and opportunities and capture relationships between these factors. These analyses were used to make more accurate predictions about future events and explore patterns in data studies that lead to better decision-making.

Furthermore Halladay (2013) also presents the evolution of predictive analysis development. The evolution began with project feasibility, which focused on project analysis and internal corporate data, continuing to be business Intelligence that already uses corporate external data integrated with internal company data and can answer why and evaluate the project from a strategic perspective. Finally, the authors decided to develop the data into Predictive Analytics, focusing on predictive modeling and forecasting tools.

Sarosa (2012); Lee and Lee (2015) predictive analytics was a process that includes a series of methodologies that can manage various large-scale data-driven problems faced by many entities. It was a repetitive process that connected several statistical methods of sampling, model estimation, model prediction, and evaluation to form a credit scoring system. According to Halladay (2013), an essential stage in developing information about predictive analytics was setting the goals of the prediction itself to determine the business problems that must be solved. While according to Lee and Lee (2015), some essential things that cannot be ignored in organizing predictive analytics work are the collection and sampling of data, model estimation, model prediction and evaluation, and model adjustments. 
Observing the credit default events in the retrofitting process in the energy efficiency industry must be understood as a flow business process. When starting the marketing process, officers look for prospective customers, the data verification/validation process, the credit analysis process, the verdict process, and the disbursement process. With the above explanation, this research was expected to provide some of the following benefits: 1) The use of machine learning in its particular retrofitting practice on granting energy efficiency project credit is a new practice and is starting to grow in Indonesia. Therefore, this research is expected to contribute to science, especially accepting technological innovation to produce efficiency in the energy efficiency industry. 2) The results of this research are expected to be one of the sources of information about retrofitting practices with Machine Learning to detect credit risk. 3) This research can be helpful to be one of the references for decision-makers in the energy efficiency industry, namely problematic credits carried out by either outsiders or parties in the provision of retrofitting credit. 4) Providing information about alternative detection models, and 5) Creating a culture in the energy efficiency industry using machine learning-based technology innovations.

\section{LITERATURE REVIEW}

Neural networks and logistics have a long history, and many individuals have worked on their development throughout the years. In recent years, many fields have created outcome prediction models based on artificial neural networks ("AN") and logistic regression analysis ("LR"). According to empirical investigations, logistic regression (LR) was the most remarkable statistical analytic technique for creditworthiness (Lessmann, Baesens, Seow, \& Thomas, 2015). The authors of this research look at ESCO's procedures for determining client creditworthiness. The authors looked at which elements influenced ESCO's final retrofitting project. To begin, the authors evaluate applications using traditional methods such as upfront investment, income factors, extra income variables, return durations, investment returns, and criteria related to client characteristics. The authors then employ Machine Learning (ML) neural networks and Logistic regression to forecast the likelihood of future behaviors (Lessmann, Baesens, Seow, \& Thomas, 2015).

In most cases, logistic regression is utilized in the credit score model. The creditworthiness model (Wiginton, 1980) used a logistic model. Cramer (2004) later created some other types of logistic regression. According to the experimental data, the border logistic regression approach has a greater classification accuracy. Overall, while the creditworthiness model performs well in machine learning, it needs big raw data. Logistic regression is one of the techniques borrowed by machine learning from statistics. It was a method for solving binary classification problems (two class values). The authors write a logistic regression algorithm for machine learning in this paper. The logistics function was also called the sigmoid function. An S-shaped curve can take an absolute value number and map it into values between 0 and 1, but never precisely at that limit. Logistic regression hypotheses tend to limit functions between $\mathrm{O}$ and ${ }_{1} 0 \leq h_{\theta}(x) \leq 1$. In machine learning, the authors use sigmoids to map probability predictions.

The authors expect the classifier to provide a set of outputs or classes based on probability when passing input through the prediction function and returning a probability score between 0 and 1 . Logistic regression becomes a probability model. In this paper, the prediction models creditworthiness stated as 'credit default' or 'default note.' The first-class can 'default,' and the logistic regression model can be written as a possible 'default' or $\mathrm{P}$ (Creditworthiness=default $\mid$ credit-score). It can be written in another way; the authors model the probability that the input $(\mathrm{X})$ belongs to the default class $(\mathrm{Y}=1)$; hence the authors can write this formally as $\mathrm{P}(\mathrm{X})=\mathrm{P}(\mathrm{Y}=1 \mid \mathrm{X})$. Logistic regression was a binary classification approach that may predict when the item has only two potential values. For example, depending on age, yearly income, height, weight, and other factors, the researchers could determine whether a person was a male (0) or female (1). Logical regression was more straightforward than a neural network.

Artificial Neural networks have been widely used for the credit-scoring problem (Lee \& Chen, 2005; West, 2000). Different ANNs have been suggested to tackle the credit scoring problem more recently. In the empirical result, the 
neural networks achieve the highest average correct classification rate compared with the LR model, considering that results were very close Abdou and Pointon (2011). When a complicated nonlinear connection between credit scoring's features was shown, the ANN model offered a considerable benefit; according to West (2000) and Hájek (2011) ANN and LR are techniques for categorization problems with a finite number of options (e.g., not a continuous value). Many people utilize the sigmoid and softmax functions to solve multiclass-classification issues in logistic regression. The structure of neural networks was relatively specific, with one input layer, at least one hidden layer, and eventually one output layer. Both ANN and LR have supervised Machine Learning algorithms with two primary goals: 1) training the model and 2) predicting it. Both algorithms face the same challenge: determining the optimum parameter value.

\section{METHOD}

In creditworthiness research, result prediction models based on artificial neural networks and logistic regression analysis have been created. Both of these strategies have benefits and drawbacks. Both of these strategies have benefits and drawbacks. This study investigated the efficacy of artificial neural networks and logistic regression models in predicting credit default occurrences in the energy efficiency industry's retrofitting. A test with a ROC value of one perfectly differentiates two outcomes, but a test with a ROC value of 0.5 was entirely worthless and performed no better than chance. This study takes the form of a case study at the ESCO firm MWS, and the authors employ a 'pseudo name' for the company's name to safeguard an institution's security. An empirical research approach was applied in this study. Empirical evidence can show the truth of a particular event using this technique. As a result, the core data for this study was based on incident data from MWS's business database.

The research focuses on MWS adopting retrofitting practices with their own ESCO model on energy management or conservative energy projects or activities. MWS was intended to introduce the latest PSS model in their own ESCO model with their capital in Indonesia. MWS conducts simple financial analysis to finance retrofitting projects to determine the appropriate project's value, risk, and liquidity effects. Energy efficiency projects need to be considered on par with others to succeed. MWS uses financial analysis to assess whether investments generate reasonable returns while not damaging the consumer base. This research was conducted intensively from July 2020 to June 2021 . Activities include research preparation, data collection, data analysis process, and reporting of research results in research conducted in Jakarta and Bekasi. The implementation of the research begins with the collection of data until the final report of the research takes \pm ten weeks.

Customer's creditworthiness and retrofitting default risks were becoming more crucial for ESCO. Therefore, upon LCC analysis, MWS was conducted to assess the cooperative's causal relationship by using the characteristics of problems in causal relationships between creditworthiness variables and other variables. Dependent variables were retrofitting feasibility, i.e., Rejected Project or Accepted Project. The independent variables, namely Initial upfront investment, revenue factors, additional revenue factors, payback period, investment returns, and customer characteristics, were the most significant factors that need to consider; see Table 2.

The process of developing the predictive model includes data access, data preparation, development of a training set, selection of an algorithm, training the model, the model testing, valuation of the model, and implementation. The simulation runs were repeated several times with different configurations to improve results. The visualization of the findings can be achieved in a variety of ways. A Logistic Regression and Artificial Neural Networks using ML have been done.

The data analysis approach was a credit score prediction model using machine learning to choose the appropriate model in this research. In this research, a definitive inference test, in addition to hypothesis testing, was also conducted. The research looked at the relationship between independent and dependent variables. The frame of mind of this research can be explained in four parts, namely identifying research and research-gap problems in the 1st stage; 
analysis of the causative factors/impact in stage 2; and problem mapping with theoretical models and discussion of results analysis in the third and fourth stages.

Table 2. Variable, membership function dan range.

\begin{tabular}{|c|c|c|c|c|}
\hline No & Aspect & Input Variable & $\begin{array}{l}\text { Membership } \\
\text { Function }\end{array}$ & Range \\
\hline 1 & Project Agreement & Agreement Type & ESA \& ESPC & $\begin{array}{l}\mathrm{ESA}=0.8-1.0 \\
\mathrm{ESPC}=0.5-0.7\end{array}$ \\
\hline 2 & $\begin{array}{l}\text { Upfront } \\
\text { Investment }\end{array}$ & $\begin{array}{l}\text { Tolerance } \\
\text { Factors }\end{array}$ & $\begin{array}{l}\text { High Risk, } \\
\text { Medium \& Low } \\
\text { Risk }\end{array}$ & $\begin{array}{l}\text { High Risk: Tolerance }>1.5 \text { Bio } \\
\text { Medium Risk: Tolerance }>750 \text { Mio } \& \\
<=1.5 \text { Bio } \\
\text { Low Risk: Tolerance }<=750 \text { Mio }\end{array}$ \\
\hline 3 & Revenue Factors & Project Margin & $\begin{array}{l}\text { High, Medium \& } \\
\text { Low }\end{array}$ & $\begin{array}{l}\text { High: Margin }>30 \% \\
\text { Medium: Margin } 10-30 \% \\
\text { Low: Margin }>10 \%\end{array}$ \\
\hline 4 & $\begin{array}{l}\text { Additional } \\
\text { Revenue Factors }\end{array}$ & Interest Loan & $\begin{array}{l}\text { High, Medium \& } \\
\text { Low }\end{array}$ & $\begin{array}{l}\text { High: Interest Loan }>\text { Time Deposit } \\
\text { Rate } \\
\text { Medium: Interest Loan = Time } \\
\text { Deposit Rate } \\
\text { Low: Interest Loan }<\text { Time Deposit } \\
\text { Rate }\end{array}$ \\
\hline 5 & Payback Period & Payback Years & $\begin{array}{l}\text { High Risk, } \\
\text { Medium \& Low } \\
\text { Risk }\end{array}$ & $\begin{array}{l}\text { High Risk: Years }>3 \text { Years } \\
\text { Medium Risk: } 1-3 \text { years } \\
\text { Low Risk: Years }<=1\end{array}$ \\
\hline 6 & Investment Return & NPV & $\begin{array}{l}\text { High, Medium \& } \\
\text { Low }\end{array}$ & $\begin{array}{l}\text { High: NPV }>30 \% \text { of Upfront } \\
\text { Investment } \\
\text { Medium: NPV } \sim 10 \%-30 \% \text { of } \\
\text { Upfront Investment } \\
\text { Low: NPV }<10 \% \text { of Upfront } \\
\text { Investment }\end{array}$ \\
\hline 7 & Customer & $\begin{array}{l}\text { Type of } \\
\text { Customer }\end{array}$ & $\begin{array}{l}\text { High Risk, } \\
\text { Medium \& Low } \\
\text { Risk }\end{array}$ & $\begin{array}{l}\text { High Risk }=1 \\
\text { Medium }=0.5-1 \\
\text { Low Risk }=0.2-0.5\end{array}$ \\
\hline
\end{tabular}

\section{RESULTS}

\subsection{Performance Evaluation Criteria}

The confusion matrix offered data for comparing the system's (model) classification results to the actual classification results. The error matrix was another name for the confusion matrix. The confusion matrix was a matrix table that described the classification model's performance on a set of test data whose actual values were known. In the confusion matrix, four terms indicate the categorization process' findings were True Positive (TP), True Negative (TN), False Positive (FP), and False Negative (FN). The following is a basic case example of predicting whether or not a customer defaults on the payment. True Positive (TP) - Data that was expected to be right was found to be true. For instance, a customer who fails on a retrofitting payment (class 1) forecasts that the customer will also default on payment (class 1). True Negative (TN) - It was projected that negative data would be actual. Customers that do not default on retrofitting payments (class 2), for example, are predicted to not default on payments by the model (class 2). Type I Error - False Positive (FP) — Negative data was forecasted as positive data. Customers who, for example, do not default on retrofitting payments (class 2) but are predicted to default on payments by a model (class 1). Type II Error: False Negative (FN) - Positive data was supposed to be negative. A customer, for example, defaults on a retrofitting payment (class 1), but the model predicts that the customer would not default on the payment (class 2).

In some cases, the Type II Error was more dangerous. We can relate that statement to the example of the credit default prediction above. If the customer does not default but was predicted to default (FP), then in the following 
prediction, ESCO can find out the actual situation that the customer did not default. However, if customers default but are predicted not to default $(\mathrm{FN})$, the company will find the actual situation very late, and ESCO will suffer financial losses. So, it can be said that Type II Error was dangerous. The confusion matrix will tell how well the model we make. In particular, the confusion matrix also provides information about TP, FP, TN, and FN. It was beneficial because the classification results generally cannot be adequately expressed in one number only. The confusion matrix allows us to see from whom to predict the credit default quickly, how much to default and not. The confusion matrix in the image below shows that companies were said to not default in the ANN - 473 companies, but 470 companies were declared not to default when using the LR model (TP). According to the LR (TN) model, the ANN model predicts 43 companies default correctly and 42 companies according to the LR (TN) model. The correct prediction (TP) was in the diagonal table in the upper left corner, so visually, it was straightforward to see the prediction error because it was in the table outside the left corner diagonal; the confusion matrix is depicted in Figure 2.
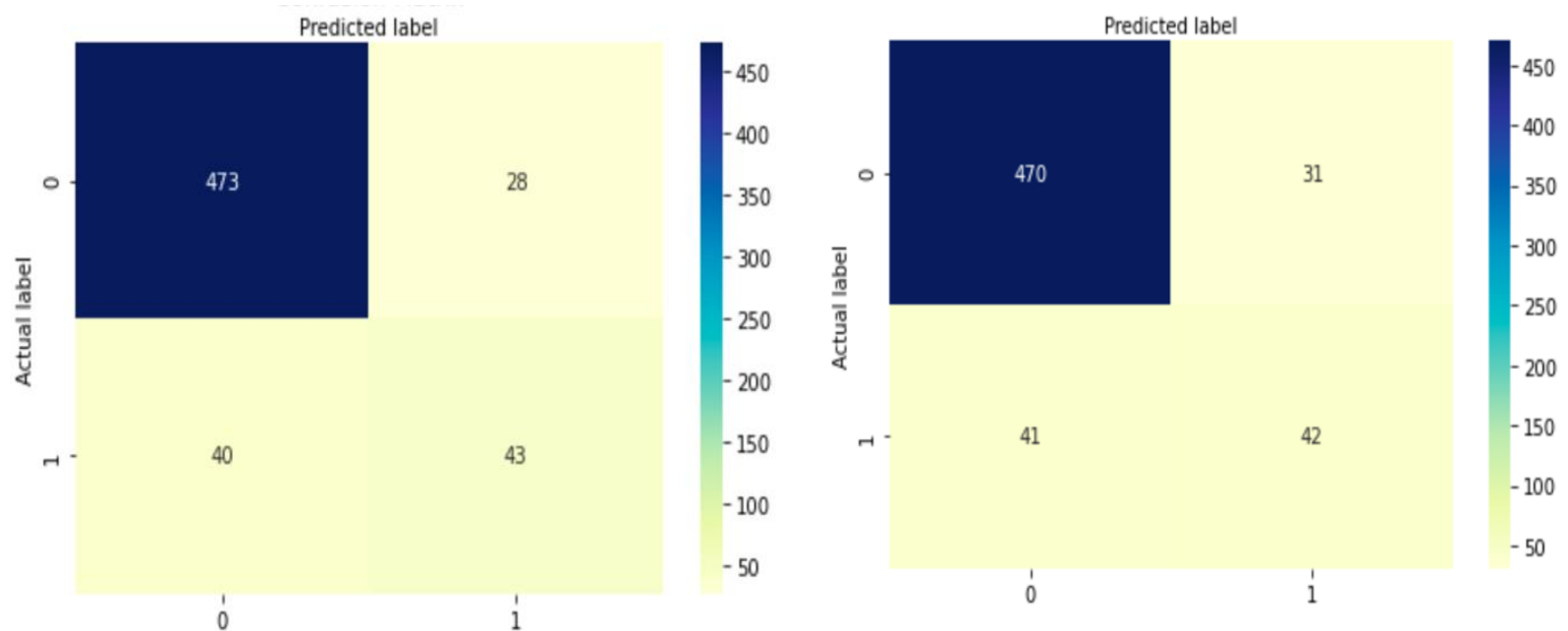

Figure 2. Confusion matrix - artificial neural network \& logit model.

The result showed that the model's accuracy was generally measured based on correctly classified instances. Both ANN and LR Models were chosen to optimize predictive accuracy. The term accuracy refers to the model's ability to categorize data correctly. As a result, accuracy was defined as the proportion of accurate predictions (both positive and negative) to the total data. To put it another way, accuracy was the degree to which the projected value was near to the actual (actual) value. The accuracy value can be obtained by equation $\mathrm{TP}=(\mathrm{TP}+\mathrm{TN}) /(\mathrm{TP}+\mathrm{TN}+\mathrm{FP}+$ FN). From the binary classification confusion matrix example above, calculating the accuracy value can answer the question, what percentage of customers were correctly predicted to default or who do not default from all customers? Based on Figure 2, the accuracy of ANN was $(473+43) /(473+43+28+40)=88.36 \%$, on the LR model was shown $(470+42) /(473+42+31+41)=87.67 \%$. The prediction rate result on the ANN model that refers to the percentage of correct predictions among all test data was as follows: True Positive Rate $(\mathrm{TPR})=473 /(473+40)=92.20 \%$, TPR or sensitivity or recall was the percentage of correctly classified cases of creditworthiness. On the LR model it shown $470 /(470+41)=91.98 \%$. True Negative Rate $(\mathrm{TNR})=43 /(43+28)=60.56 \%$, TNR or specificity was the percentage of correctly classified cases. On the LR model, it shown $42 /(42+31)=57.53 \%$. TPR was the number of positive examples classified as positive, and TNR was the number of negative examples classified as negative.

On the other hand, precision refers to the degree of agreement between the desired data and the model's predicted outputs. Consequently, precision was defined as the ratio of accurate positive predictions to correct positive expected outcomes. Of all the positive classes that have been correctly predicted, how many data were truly positive. The TP / $(\mathrm{TP}+\mathrm{FP})$ equation can obtain the precision value. From the binary classification confusion matrix example above, 
calculating the precision value can answer the question, what percentage of customers were correct to default out of all customers predicted to default? The answer for the precision rate of ANN were $473 /(473+28)=94.41 \%$ and on LR model $470 /(470+31)=93.81 \%$.

Based on Lessmann et al. (2015) the authors need to build evaluation criteria to quantify the misclassification of default assessment models. This article utilized this relative ratio to create a cost indicator that indicates the credit risk assessment model's misclassification cost. We also constructed the cost of credit risk as False Negative Rate $(\mathrm{FNR})=\mathrm{FN} /(\mathrm{FN}+\mathrm{TP})=40 /(40+743)=7.80 \%$ and False Positive Rate $(\mathrm{FPR})=\mathrm{FP} /(\mathrm{FP}+\mathrm{TN})=28 /(28+43)$ $=5.59 \%$. On the other hand, FNR and FPR on the LR model were $8.02 \%$ and $6.19 \%$, respectively. FNR was the number of positive examples classified as negative (i.e., type I error), and FPR was the number of negative examples classified as positive (i.e., type II error). Lessmann reported that the ratio of misclassification costs associated with type II and type I errors was 5:1, which (Abdou, 2009) used. Hence the cost of credit risk become $=5 \times(7.80 \%+$ $5.59 \%$ ) or $66.93 \%$ based on ANN model and $71.06 \%$ based on LR model.

A ROC curve (receiver operating characteristic curve) showed the classification performance. The Receiver Operating Characteristics (ROC) curve, which shows the false positive rate (FPR) on the Y-axis versus the true positive rate (TPR) on the $\mathrm{X}$-axis over a range of threshold values, was formerly a standard technique.
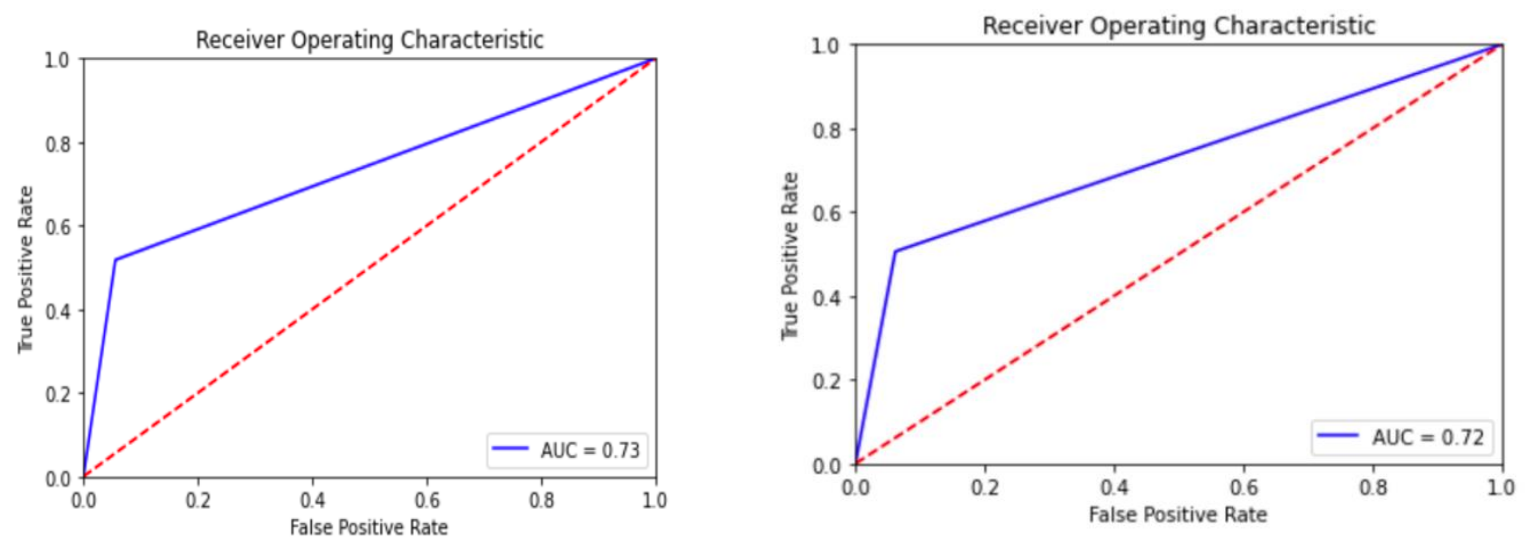

Figure 3. ROC curve - artificial neural network \& logit model.

Simultaneously, the ANN algorithm-based model showed the best result under the ROC Curve metric in the other area. The ideal ROC curve is parallel to the Y-axis, which is impossible to accomplish in practice. The model that comes closest to it will be the best. Under the ROC Curve Value (AUROC), a number between 0 and 1 was traditionally used to summarize the ROC curve. The better the model, the greater the AUROC. AUC was a composite measure of success that considers all possible classification thresholds. The model's likelihood rates a random positive example higher than a random negative example was one way to view AUC. A receiver operating characteristic curve (ROC curve) was a graph that shows how well a classification model performs overall classification thresholds. Two parameters were plotted on this curve. TPR vs. FPR was plotted on a ROC curve at various classification thresholds. Lowering the classification threshold causes more things to be classified as positive, resulting in more False Positives and True Positives. A typical ROC curve for ANN was 0.73, and LR was at 0.72, shown in Figure 3.

\subsection{Creditworthiness Success Analysis}

Logistic regression was a subset of a neural network classifier. The researchers can simulate a logistic regression model using a neural network with one hidden node with the identity activation function and one output node with zero bias and logistic sigmoid activation. In principle, anything researchers can do with logistic regression with a neural network. Therefore, theoretically, a neural network was always better than logistic regression, or more precisely, a neural network can do no worse than logistic regression. The ESCO was more worried about elements 
that enhance the likelihood of successful retrofitting than whether the model correctly forecasts the probability of credit risk scoring. This conclusion was made under the methods used by Liang and He (2020) and Cai et al. (2016) to assess the success of credit risk and loan prediction models. Thus we picked the logistic regression model over a neural network in this part. In addition, this section investigates all factors that influence the likelihood of effective retrofitting. Table 3 showed the results of the model's p-value was $1,852 \times 10^{\wedge}-187$, which was less than 0.01 or $1 \%$ as was thought 'excellent. The $\mathrm{p}=$ value of each variable as a list under $\mathrm{P}>|\mathrm{z}|$ was less than 0.01 or $1 \%$. Pseudo RSquared was 0.4810 or $48.19 \%$. From the pseudo-R-Square value, all independent variables' influence on dependent variables (retrofitting project) was $48.19 \%$. Obtained multinomial logistics regression model for log category ratio retrofitting project $=1$ (Take) and retrofitting project $=0$ (Hold and Reject) was $-6.5619+-4.0780$ (Project Agreement) + 0.5237 (Upfront Investment) + 2.0639 (Revenue Factors) + 0.4995 (Additional Revenue Factors) + 0.5579 (Investment Return) + 1.3236 (Customer) - 0.7805 (Type of Customer)

Table 3. Logistic results.

\begin{tabular}{|c|c|c|c|c|c|c|}
\hline EXPERT_JUDGE=0=1 & Coeff & Std. Error & $\mathbf{Z}$ & $\mathbf{P}>|\mathbf{z}|$ & {$[0.025]$} & {$[0.975]$} \\
\hline Constant & $(6.562)$ & 0.694 & $(9.445)$ & 0.000 & $(7.992)$ & $(5.202)$ \\
\hline Project agreement & $(4.078)$ & 0.280 & $(14.546)$ & 0.000 & $(4.627)$ & $(3.529)$ \\
\hline Upfront investment & 0.524 & 0.104 & 5.058 & 0.000 & 0.321 & 0.727 \\
\hline Revenue factors & 2.064 & 0.139 & 14.826 & 0.000 & 1.791 & 2.337 \\
\hline Additional revenue factors & 0.500 & 0.105 & 4.768 & 0.000 & 0.294 & 0.705 \\
\hline Investment return & 0.558 & 0.104 & 5.366 & 0.000 & 0.354 & 0.762 \\
\hline Customer & 1.324 & 0.118 & 11.255 & 0.000 & 1.093 & 1.554 \\
\hline Type of customer & $(0.781)$ & 0.170 & $(4.603)$ & 0.000 & $(1.113)$ & $(0.448)$ \\
\hline
\end{tabular}

\section{CONCLUSION}

This research briefly compares logistic regression and artificial neural networks with shared roots in statistical pattern recognition. Predictive modeling for creditworthiness detection was done in this report. The model was built on the LR and ANN model of machine learning. The model was developed and tested using the Python algorithm, and the proposed model's efficiency was demonstrated. The logistic regression calculations showed that the accuracy value of prediction data with test data was $88.3562 \%$ and $87.67 \%$ for ANN and LR models. The prediction rate result that refers to the correct predictions among all test data for ANN and LR model was $92.20 \%$ and $91.98 \%$, respectively. The percentage of customers who were correct to the default of all customers predicted to default were $94.41 \%$ for ANN and $93.81 \%$ for the LR model. This case study reveals that the ANN model was better than the LR model, especially when a complex nonlinear relationship was presented between credit features. It was in line with the other empirical research that a neural network was always better than logistic regression. More precisely, a neural network can do no worse than logistic regression.

On the other hand, the vast opportunities in retrofitting projects come with significant risks. The biggest issue was that the company was hampered by a lack of knowledge about the clients' reputation. The customer's creditworthiness was critical to ESCO's survival. The project's numerous factors, particularly the qualitative customer characteristic, were vital factors to consider. As a result, ESCO companies increasingly rely on machine learning techniques. The research aimed to determine how to construct a predictive model using machine learning techniques and whether it would be easy, convenient, and accessible for non-experts. We found that the machine learning methods for creditworthiness prediction in retrofitting projects were fresh and worth a shot. Machine learning used the logistic regression model's predictive efficiency by pre-processing data using ANN and LR models. It was hoped that this new practice would grow in popularity and become standard among ESCOs. Unfortunately, current machine-learning-based creditworthiness scoring practices lack explainability and interpretability. In terms of the credit approval process, most of these algorithms are called black boxes. Under the circumstances, ESCO must penalize the retrofitting project. As a result, since retrofitting was a new industry, the credit approval process was 
challenging to communicate to consumers. The most important thing for ESCO to deal with the project was friendship and know-how with the client.

Predictive modeling for creditworthiness detection was done in this report. The model was built on the LR and ANN models of machine learning. The model was developed and tested using the Python algorithm, and the proposed model's efficiency was demonstrated. Some of the limitations of this research related to creditworthiness prediction research were based on case studies and limited to ESCO companies, namely MWS. The prediction was reflected in the scoring value that can be an Early Warning Signal (EWS) tool for managers in 'MWS' companies in mitigating bad credit risk. The other limitation was that this research related to creditworthiness scoring with various industries was more varied considering the different business processes and models. This research was based on the energy efficiency industry, especially in the case study of MWS companies. Moreover, the research was finally limited to the effectiveness of efforts to prevent credit jams with alternative approaches compared to traditional approaches on MWS companies.

Funding: This study received no specific financial support.

Competing Interests: The authors declare that they have no competing interests.

Authors' Contributions: All authors contributed equally to the conception and design of the study.

\section{REFERENCES}

Abdou, H. A., \& Pointon, J. (2011). Credit scoring, statistical techniques and evaluation criteria: A review of the literature. Intelligent Systems in Accounting, Finance and Management, 18(2-3), 59-88.Available at: https://doi.org/10.1002/isaf.325.

Abdou, H. A. P. J. (2009). Intelligent systems in accounting, finance, and management. Intelligent Systems in Accounting, Finance, and Management, 16(1-2), 21-31.

Angelis, D. I., \& Nussbaum, D. (2015). Cost analysis. Military Cost-Benefit Analysis: Theory and Practice, $113-135$.

Cai, D. J., Aharoni, D., Shuman, T., Shobe, J., Biane, J., Song, W., . . . Lou, J. (2016). A shared neural ensemble links dis tinct contextual memories encoded close in time. Nature, 534(7605), 115-118.Available at: https://doi.org/10.1088/17412552 /ab2ffa.

Cramer, J. A. (2004). A systematic review of adherence with medications for diabetes. Diabetes Care, $27(5), 1218-1224$.

Dobbs, R., Pohl, H., Lin, D.-Y., Mischke, J., Garemo, N., Hexter, J., \& Nanavatty, R. (2013). Infrastructure productivity: How to save $\$ 1$ trillion a year: McKinsey Global Institute (Issue January).

Fornara, F., Pattitoni, P., Mura, M., \& Strazzera, E. (2016). Predicting intention to improve household energy efficiency: The role of value-belief-norm theory, normative and informational influence, and specific attitude. Journal of Environmental Psychology, 45, 1-10.Available at: https://doi.org/10.1016/j.jenvp.2015.11.001.

Frankel, D., Heck, S., \& Tai, H. (2013). Sizing the potential of behavioral energy-efficiency initiatives in the US residential market: McKinsey \& Company.

Fuller, S. K., \& Petersen, S. R. (1996). LC costing manual for the federal energy management program. NIST Handbook, 135.

Hájek, P. (2011). Municipal credit rating modelling by neural networks. Decision Support Systems, 51(1), 108-118.Available at: https://doi.org/10.1016/j.dss.2010.11.033.

Halladay, B. S. D. (2013). Using predictive analytics to improve decision making. Journal of Equipment Lease Financing, 31 (2), 1-6. Heesen, F., \& Madlener, R. (2016). Technology acceptance as part of the energy performance gap in energy- efficient retrofitted dwellings Florian Heesen and Reinhard Madlener December 2014 Revised February 2016 Institute for Future Energy Consumer Needs and Behavior ( FCN ) (FCN Working Paper No. 25/2014; Issue 25).

Husin, N. I., Ahmad, A. C., Ab Wahid, A. M., \& Kamaruzzaman, S. N. (2017). Energy efficiency criteria for green highway. Social Science Eं Humanities, 25(S), 115-124.

Kumbaroğlu, G., \& Madlener, R. (2012). Evaluation of economically optimal retrofit investment options for energy savings in buildings. Energy and Buildings, 49, 327-334.Available at: https://doi.org/10.1016/j.enbuild.2012.02.022. 
Lee, T.-S., \& Chen, I.-F. (2005). A two-stage hybrid credit scoring model using artificial neural networks and multivariate adaptive regression splines. Expert Systems with Applications, 28(4), 743-752.

Lee, I., \& Lee, K. (2015). The internet of things (IoT): Applications, investments, and challenges for enterprises. Business Horizons, 58(4), 43 1-440.Available at: https://doi.org/10.1016/j.bushor.2015.03.008.

Lessmann, S., Baesens, B., Seow, H.-V., \& Thomas, L. C. (2015). Benchmarking state-of-the-art classification algorithms for credit scoring: An update of research. European Journal of Operational Research, 247(1), 124-136.Available at: https://doi.org/10.1016/j.ejor.2015.05.030.

Liang, K., \& He, J. (2020). Analyzing credit risk among Chinese P2P-lending businesses by integrating text-related soft information. Electronic Commerce Research and Applications, 40, 100947.Available at: https://doi.org/10.1016/j.elerap.2020.100947.

McWilliams, J. A., \& Walker, I. S. (2005). Home energy article: A systems approach to retrofitting residential HVAC systems: Lawrence Berkeley National Laboratory (Issue April).

Ruparathna, R., Hewage, K., \& Sadiq, R. (2017). Economic evaluation of building energy retrofits: A fuzzy based approach. Energy and Buildings, 139, 395-406.Available at: https://doi.org/10.1016/j.enbuild.2017.01.031.

Sarosa, S. (2012). Adoption of social media networks by Indonesian SME: A case study. Procedia Economics and Finance, 4, 244254.Available at: https://doi.org/10.1016/s2212-5671(12)00339-5.

Stern, P. C. (2011). Contributions of psychology to limiting climate change. American Psychologist, 66(4), 303-314.

The United States Environmental Protection, A. (2008). Energy star ${ }^{\circledR}$ Building upgrade manual energy star ${ }^{\circledR}$ Building Upgrade Manual Contents (Office of Air and Radiation 2008 Edition).

West, D. (2000). Neural network credit scoring models. Computers \& Operations Research, 27(11-12), 1131-1152.

Wiginton, J. C. (1980). University of washington school of business administration Cambridge University Press. Journal of Financial and Quantitative Analysis, 15(3), 757-770.

WSGR. (2015). Innovative financing for renewable energy innovative financing for renewable energy ARTICLE innovative financing for renewable energy. Retrieved from: https://digitalcommons.pace.edu/pelr.

Views and opinions expressed in this article are the views and opinions of the author(s), Financial Risk and Management Reviews shall not be responsible or answerable for any loss, damage or liability etc. caused in relation to/arising out of the use of the content. 